\title{
DETECTION OF DIABETIC MACULOPATHY IN HUMAN RETINAL IMAGES USING MORPHOLOGICAL OPERATIONS
}

\author{
${ }^{1}$ G.S. Annie Grace Vimala and ${ }^{2}$ S. Kajamohideen \\ ${ }^{1}$ ECE, St. Joseph's Institute of Technology, Chennai, India \\ ${ }^{2}$ ECE, B.S. Abdur Rahman University, Chennai, India
}

Received 2014-06-28; Revised 2014-07-04; Accepted 2014-07-14

\begin{abstract}
Diabetic Retinopathy (DR) is a common retinal complication associated with diabetes. Adverse changes in the retinal blood vessels lead to loss of vision without any symptoms. Diabetic retinopathy is frequent, blinding complication of the patient with high blood sugar levels that characterize diabetes. Automatic recognition of DR lesions like exudates, in digital fund us images can contribute to the diagnosis and screening of this disease. The Exudates present in the macula which is the center portion of the retina is called maculopathy or macular edema. In this approach, an automatic and efficient method to detect diabetic maculopathy which is a severe stage of diabetic retinopathy, is proposed. The real time retinal images were obtained from a nearby hospital. The retinal images were pre-processed via. Contrast Limited Adaptive Histogram Equalization (CLAHE). The preprocessed color retinal images are subjected to top hat transform and bottom hat transform. The Macula which is the darkest region was obtained. To classify the preprocessed image into exudates and non-exudates, a set of features based on color and texture were extracted. Classification was done using support Vector Machine This method appears promising as it can detect the severity of the disease.
\end{abstract}

Keywords: Diabetic Retinopathy, Maculopathy, Fundus Image, Morphological Operations, SVM

\section{INTRODUCTION}

World Health Organization (WHO) predicts that developing countries will bear the brunt of the epidemic in the 21st century (Quellec et al., 2011). An estimated 285 million people, corresponding to $6.4 \%$ of the World's adult population, will live with diabetes at the end of 2015 (Osareh et al., 2009). The number is expected to grow to 438 million by 2030 ,corresponding to $7.8 \%$ of the adult population. $70 \%$ of the current cases of diabetes occur in low and middle income countries. Agurto et al. (2010) With an estimated 50.8 million people living with diabetes, India has the world's largest diabetes population, followed by China with 43.2 million. $80 \%$ of all patients who have diabetes for 10 years or more or prone to diabetic retinopathy. There are two main mechanisms by which vision is lost due to diabetic retinopathy: Macular edema and proliferative retinopathy. Macular edema is the accumulation of fluid called exudate in macula. This is the main cause of vision loss and its prevalence is set to continue rising. The retina is the inner and most important layer of the eye. It is composed of several important anatomical structures which can indicate various diseases. Cardiovascular disease such as stroke and myocardial infarcation can be identified from retinal blood vessels. diabetic retinopathy is the common retinal complication associated with diabetes. It is a major cause of blindness in both middle and advanced age groups.

Exudates are one of the primary signs of diabetic retinopathy (Sopharak et al., 2009). Exudates are yellowwhite lesions with relatively distinct margins. Exudates are lipids and proteins that deposits and leaks from the damaged blood vessels within the retina. Detection of exudates by ophthalmologists is a laborious process as

Corresponding Author: G.S. Annie Grace Vimala, ECE, St. Joseph's Institute of Technology, Chennai, India 
they have to spend a great deal of time in manual analysis and diagnosis. Moreover, manual detection requires using chemical dilation material which takes time and has negative side effects on the patients. Hence automatic screening techniques for exudates are preferred.

\subsection{Overview of State of Art}

Osareh et al. (2009) proposed a method for automatic identification of exudates based on computational Intelligence technique. The colour retinal images were segmented using fuzzy c-means clustering. Feature vector were extracted and classified using multilayer neural network classifier. Walter et al. (2002) identified exudates from green channel of the retinal images according to their gray level variation. The exudates contour was determined using mathematical morphology techniques. However the author ignored some types of errors on the border of the segmented exudates in their reported performances and did not discriminate exudates from cotton wool spots. Sopharak et al. (2009; Niemeijer et al., 2007a) reported the result of an automated detection of exudates from low contrast digital images of retinopathy patients with non-dilated pupils by fuzzy c-means clustering. Four features such as intensity, standard deviation on intensity, hue and a number of edge pixels were extracted and applied as input to coarse segmentation using FCM clustering method. Niemeijer et al. (2007a; 2007b) distinguished the bright lesion like exudates, cotton wool spots and drusen from colour retinal images. In the first step, pixels were classified, resulting in a probability map that included the probability of each pixel to be part of a bright lesion.

\section{IMAGING AND IMAGE ACQUISITION}

The present study creates the awareness about diabetic retinopathy abnormality within the framework of public health. A free eye screening camp was conducted in a leading eye care hospital, Chennai, India, at the end of the year 2012. This study was performed on 50 South Indian subjects whose age ranged from 50-85 years. Prior to the study, a detailed questionnaire was filled in by the patients, signed and informed consent form to the investigation protocol. For all the subjects, digital images of size $640 \times 480$ were taken with a CARL ZEISS FF 450 plus Visupac fundus camera.

\subsection{Algorithm to Detect Diabetic Maculopathy}

Step $1=$ Get the input image

Step 2 = Obtain the green component of the image $(\mathrm{G})$ from the original RGB image

Step 3 =Apply median filter and contrast limited adaptive histogram equalization to the green component of the image

Step 4 =Apply top-hat transform to the image $G$ and add the result with the image $G$. Let the output image be $\mathrm{T} 1$

Step 5 =Apply bottom-hat transform to the image $\mathrm{G}$

Step $6=$ Subtract the result with the image T1

Step 7 Macula, which is the darkest region of an image is detected

Step $8=$ End the process

The flow diagram for diabetic maculopathy is shown in Fig. 1. The input retinal image is acquired from the Diabetic Patients. The input image is in RGB color space. From the input image, the green component alone is extracted since it contains more information about the darkest region. It is then applied to the median filter to remove the noise. The image is enhanced using contrast limited adaptive histogram equalization. The histogram equalized image is then subjected to the top-hat transform. The input image and the green component of the image are shown in Fig. 2 and 3.

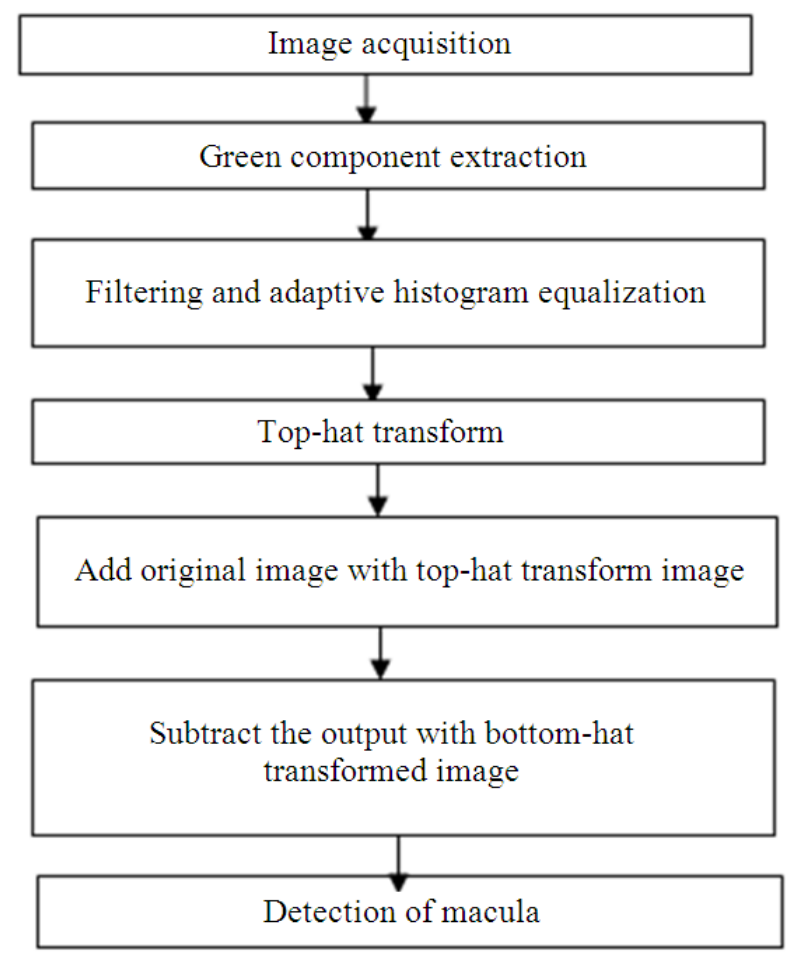

Fig. 1. Flow diagram for diabetic maculopathy 


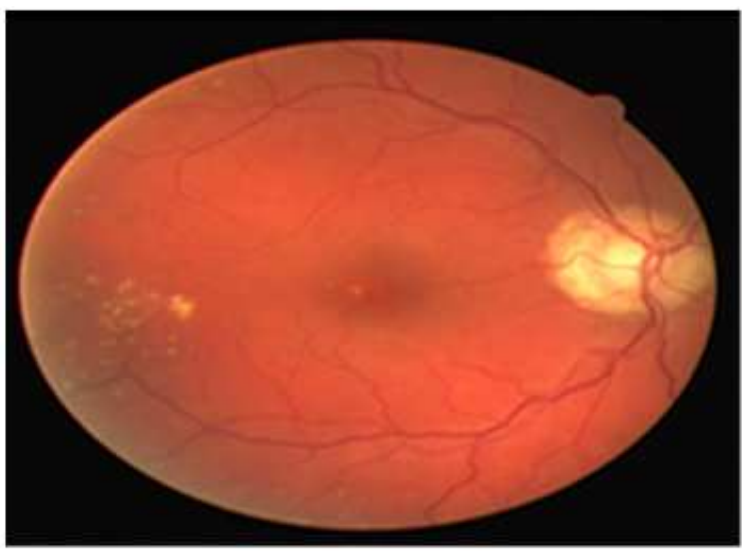

Fig. 2. Input retinal image

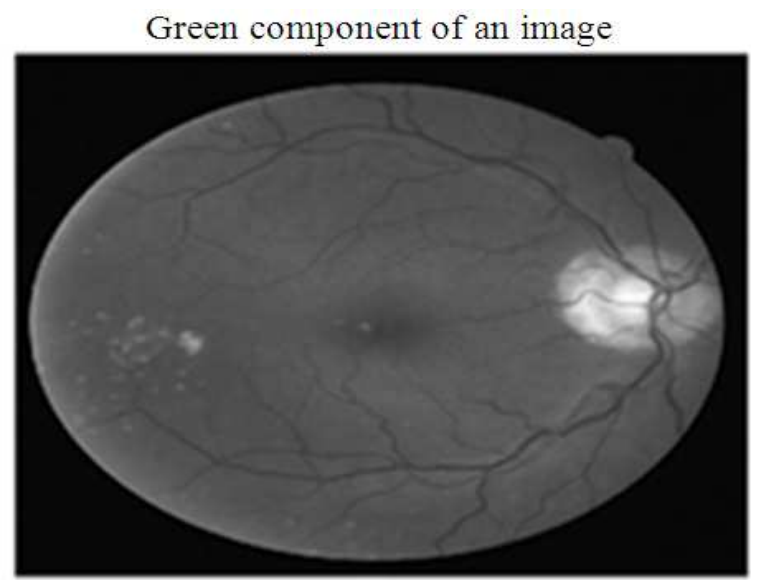

Fig. 3. Green component of an input retinal image

\subsection{Contrast Enhancement}

Colour fundus images often show important lighting variation, poor contrast and noise. In order to reduce these imperfections (Aquino et al., 2011) and generate images more suitable for extracting the pixel features in the classification process, a preprocessing comprising the following step is applied. (1) RGB to HSI conversion (2) Median filtering (3) Contrast Limited Adaptive Histogram Equalization (CLAHE).

\subsubsection{RGB to HSI Conversion}

The input retinal images in RGB Colour space are converted to HSI colour space. The noises in the images are due to the uneven distribution of the Intensity (I) component (Sanchez et al., 2006).

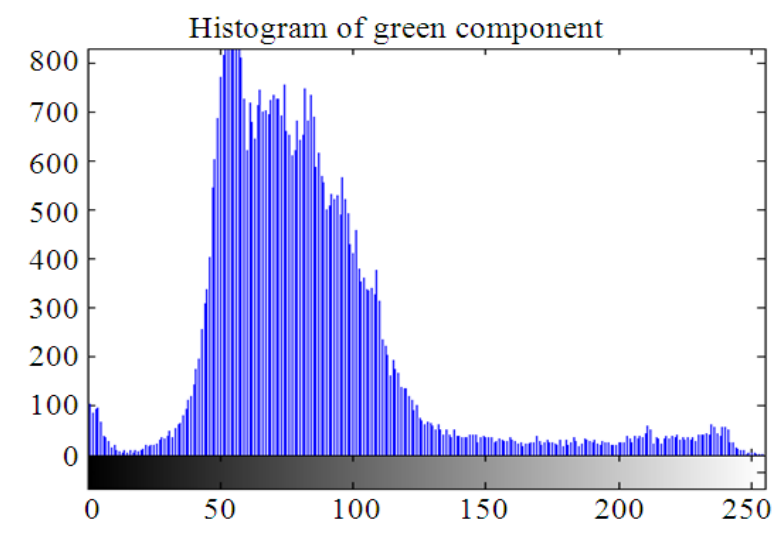

Fig. 4. Histogram of green component

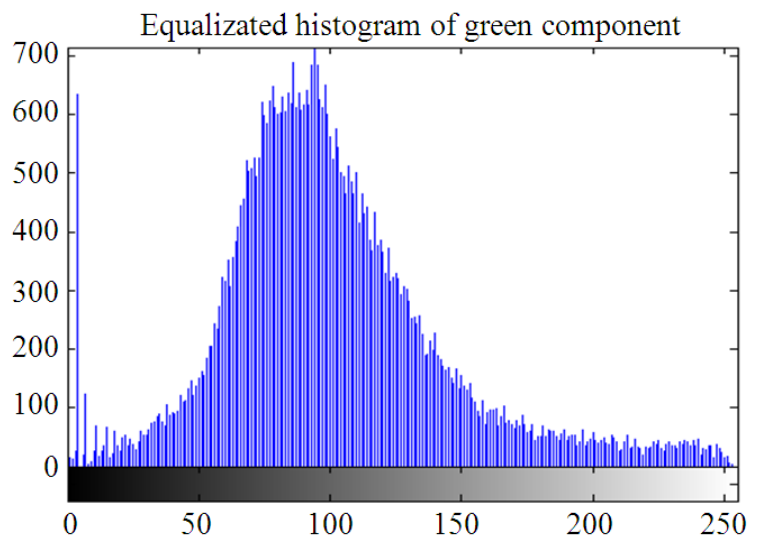

Fig. 5. CLAHE-green component

\subsubsection{Median Filtering}

In order to distribute the intensity throughout the image uniformly, the I-component of HSI colour space is extracted and filtered out through a $3 \times 3$ median filter.

\subsubsection{Contrast Limited Adaptive Histogram Equalization (CLAHE)}

The contrast limited adaptive histogram equalization is applied on the filtered I-component of the image (Fleming et al., 2007). Figure 4 and 5 shows the green component output and CLAHE output. The histogram equalized I component is combined with HS component and transformed back to the original RGB colour space.

\section{MORPHOLOGICAL OPERATION}

In this section, the basic operations of dilation, erosion, opening and closing to gray-scale images are discussed, which is used to detect the white and black regions in the 
image. Diabetic Maculopathy is a white region which is detected by the above operations. Assume that $f(x, y)$ is a gray-scale image and $b(x, y)$ is a Structuring Element (SE) and both functions are discrete. Similar to binary morphology (Fleming et al., 2007), the structuring elements are used to examine a given image for specific properties. SE belongs to one of two categories.

None flat (continuous variation of intensity rarely used) and flat. The origin of SE must be specified. Unless mentioned otherwise, SEs is flat, symmetrical, of unit height, with the origin at the center.

\subsection{Erosion}

The erosion of $f$ by a flat structuring element $b$ at any location $(x, y)$ is defined as the minimum value of the image in the region coincident with $b$ when the origin of $b$ is at $(x, y)$. Therefore, the erosion at $(x, y)$ of an image $f$ by a structuring element $b$ is given by Equation 1 :

$$
[f b](x \Theta y)=_{(s, t) s b}\{f(x+s, y+t)\}
$$

where, similar to the correlation, $x$ and $y$ are incremented through all values required so that the origin of $b$ visits every pixel in $f$. That is, to find the erosion of $f$ by $b$, the origin of the structuring element is placed at every pixel location in the image. The erosion is the minimum value of $f$ from all values of $f$ in the region of $f$ coincident with $b$. Since gray-scale erosion with a flat SE computes the min intensity value of $f$ in every neighbourhood, the eroded gray scale image should be darker (bright features are reduced, dark features are thickened and background is darker).

\subsection{Opening and Closing}

The dilation of $f$ by a flat structuring element $b$ at any location $(x, y)$ is defined as the maximum value of the image in the window outlined by $b$ when the origin of $b$ is at $(x, y)$. That is Equation 2:

$$
[f b](x \Theta y)=\max _{(s, t) s b}\{f(x-s, y-t)\}
$$

The explanation is similar to the one for erosion except for using maximum instead of minimum and that the structuring element is reflected about the origin. Since grayscale dilation with a flat SE computes the min intensity value of $f$ in every neighbourhood, the dilated gray scale image should be brighter. (Darker features are reduced, bright features are thickened and background is brighter).

\subsection{Opening and Closing}

Same as for binary images, the opening of the image $f$ by structuring element $b$ is defined as the erosion of $f$ by $b$ followed by a dilation of the result with $b$ Equation 3:

$$
f \Theta b=(f \Theta b) \oplus b
$$

Similarly, the closing of $f$ by $b$ is Equation 4:

$$
f \Theta b=(f \oplus b) \Theta b
$$

Opening: The intensity of all bright features decreased, depending on the sizes of features compared to the SE. Unlike the erosion, opening has negligible effect on the dark features and the effect on the background is negligible.

Closing: Dark features are attenuated and the background is unaffected.

\subsection{Top-Hat and Bottom Hat Transform}

Combining image subtraction with openings and closings results in top-hat and bottom-hat transformations. Top-hat transform is an operation that extracts small elements and details from given images. The top-hat transform is defined as the difference between the input image and its opening by some structuring element. Tophat transform can also be used to correct uneven illumination when the background is dark.

\section{Top-hat $=$ Original image-opened image}

The bottom-hat transform is defined as the difference between the input image and its closing by some structuring element.

Bottom-hat $=$ Original image-closed image.

One principal application of these transforms is that of removing objects from an image using an SE in the opening and objects from an image by using an SE in the opening and closing that does not fit the objects to be removed. The difference then yields an image with only the removed objects. The top-hat is used for light objects on a dark background and the bottom-hat for dark objects on a light background. An important use of top-hat transformation is correcting the effects of non-uniform illumination.

\section{FEATURE EXTRACTION}

From Table 1, sensitivity, specificity are chosen as measurement of accuracy and are calculated using the following Equation 5 to 7 (Annie Grace Vimala and Kajamohideen, 2014)7:

$$
\begin{aligned}
& \text { Sensitivity }=T P /(T P+F P) \\
& \text { Specificity }=T N /(T N+F P)
\end{aligned}
$$


Accuracy $=(T P+T N) /(T P+T N+F N+F P)$

Table 2 shows the performance analysis for different images. The sensitivity, specificity, accuracy were calculated and an average accuracy of $94.67 \%$ is obtained.

\subsection{Classification Using Support Vector Machine (SVM)}

The standard SVM is a binary classifier which has found widespread use in pattern recognition problems such as image and audio recognition, hand writing recognition, medicine, science, finance and so on. The support vector machine or SVM framework is currently the most popular approach for "off-the-shelf" supervised learning.

There are three properties that make SVMs attractive.

SVMs construct a maximum margin separator-a decision boundary with the largest possible distance to example points (Ricci and Perfetti, 2009). This helps them generalize well.

SVMs create a linear separating hyper plane, but they have the ability to embed the data into a higher-dimensional space, using the so-called kernel trick. Often, data that are not linearly separable in the original input space are easily separable in the higher dimensional space.

The high-dimensional linear separator is actually nonlinear in the original space. This means the hypothesis space is greatly expanded over methods that use strictly linear representations.

SVMs are a nonparametric method-they retain training examples and potentially need to store them all. On the other hand, in practice they often end up retaining only a small fraction of the number of examplessometimes as few as a small constant time the number of dimensions. Thus SVMs combine the advantages of nonparametric and parametric models: They have the flexibility to represent complex functions, but they are resistant to over fitting. The input points are mapped to a high dimensional feature space, where a separating hyper-plane can be found. The algorithm is chosen in such a way as to maximize the distance from the closest patterns, a quantity which is called the margin. SVMs are learning systems designed to automatically trade-off accuracy and complexity by minimizing an upper bound on the generalization error. In a variety of classification problems, SVMs have shown a performance which can reduce training and testing errors, thereby obtaining higher recognition accuracy (Annie Grace Vimala and Kaja Mohideen, 2012). SVMs can be applied to very high dimensional data without changing their formulation.

\section{RESULTS AND DISCUSSION}

Figure 6 shows the detection of diabetic maculopathy in a single GUI window. Macula is the center part of the retina (Sanchez et al., 2006). It is also the darkest part in the retinal image. This region is localized using morphological operation.

Table 1. Maculopathy classification

\begin{tabular}{lll}
\hline Exudate & $\begin{array}{l}\text { Exudate } \\
\text { Present }\end{array}$ & $\begin{array}{l}\text { Exudate } \\
\text { absent }\end{array}$ \\
\hline Maculopathy detected & True & False \\
& Positive (TP) & $\begin{array}{l}\text { Positive (FP) } \\
\text { Maculopathy not detected }\end{array}$ \\
& $\begin{array}{l}\text { False Negative } \\
\text { (FN) }\end{array}$ & $\begin{array}{l}\text { True Negative } \\
\text { (TN) }\end{array}$ \\
\hline
\end{tabular}

Table 2. Performance analysis

\begin{tabular}{lccr}
\hline Image & Sensitivity & Specificity & Accuracy \\
\hline 1 & 0.7778 & 0.9734 & 0.9577 \\
2 & 0.7665 & 0.9793 & 0.9474 \\
3 & 0.7225 & 0.9711 & 0.9649 \\
4 & 0.7032 & 0.9855 & 0.9479 \\
5 & 0.6815 & 0.9872 & 0.9459 \\
6 & 0.6296 & 0.9893 & 0.9585 \\
7 & 0.6780 & 0.9861 & 0.9453 \\
8 & 0.5704 & 0.9917 & 0.9688 \\
9 & 0.6530 & 0.9882 & 0.9489 \\
10 & 0.6967 & 0.9846 & 0.9502 \\
\hline
\end{tabular}

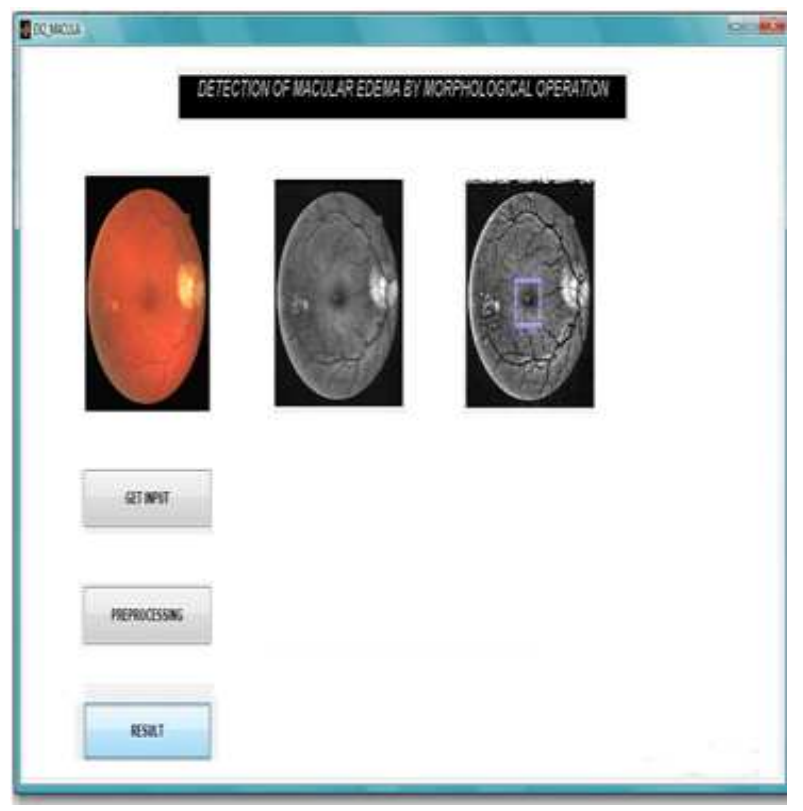

Fig. 6. Detection of macular edema 


\section{CONCLUSION}

Diabetic Maculopathy, is a severe stage of diabetic retinopathy (Shobha and Rajeshwari, 2014). This study is focused to detect the severity of the disease which is called maculopathy. The low contrast digital image is enhanced using Contrast Limited Adaptive Histogram Equalization (CLAHE). The color fundus images are subjected to pre-processing followed by edge detection using morphological operations. The overall sensitivity, specificity and accuracy is obtained as 70.42, 98.43 and $94.67 \%$ respectively. Also the detection of diabetic maculopathy, which is the severe stage of diabetic retinopathy, is obtained using morphological operation. This system is very useful in detecting the severe as well as early stage of diabetic retinopathy. The SVM classifier gives the accuracy of $94.67 \%$.

\section{REFERENCES}

Agurto, C., E. Barriga, S. Murillo, M. Pattichis and H. Davis et al., 2010. Multiscale AM-FM methods for diabetic retinopathy lesion detection. IEEE Trans. Med. Imag., 29: 502-512. DOI: 10.1109/TMI.2009.2037146

Annie Grace Vimala, G.S. and S. Kaja Mohideen, 2012. An efficient approach for detection of exudates in diabetic retinopathy images using clustering algorithm. IOSR J. Comput. Eng., 2: 43-48.

Annie Grace Vimala, G.S. and S. Kajamohideen, 2014. Diagnosis of diabetic retinopathy by extracting blood vessels and exudates using retinal color fundus images. WSEAS Trans. Biol. Biomed., 11: 20-28.

Aquino, A., M.E. Gegundez-Arias and D. Marin, 2011. Detecting the optic disc boundary in digital fundus images using morphological, edge detection and feature extraction technique. IEEE Trans. Med. Imag., $\quad 29$ : 1860-1869. DOI: 10.1109/TMI.2010.2053042

Fleming, A.D., S. Philip, K.A. Goatman, G.J. Williams and J.A. Olson et al., 2007. Automated detection of exudates for diabetic retinopathy screening. J. Phys. Med. Bio., 52: 7385-7396. PMID: 18065845

Niemeijer, B.V., B. Ginneken, S.R. Russell, M.S. Suttorp-Schulten and M.D. Abràmoff, 2007a. Automated detection and differentiation of drusen, exudates and cotton wool spots in digital color fundus photographs for diabetic retinopathy diagnosis. Invest. Ophthalmol Vis. Sci., 48: 22602267. PMID: 17460289
Niemeijer. M., M.D. Abramoff and B. Van Ginneken, 2007b. Information fusion for diabetic retinopathy $\mathrm{CAD}$ in digital color fundus photographs. IEEE Trans. Med. Imag., 28: 775-785. DOI: 10.1109/TMI.2008.2012029

Osareh, A., B. Shadgar and R. Markham, 2009. A computational-intelligence-based approach for detection of exudates in diabetic retinopathy images. IEEE Trans. Inform. Tech. Biomed., 13: 535-545. DOI: 10.1109/TITB.2008.2007493

Quellec, G., S.R. Russell and M.D. Abramoff, 2011. Optimal filter framework for automated, instantaneous detection of lesions in retinalimages. IEEE Trans. Med. Imag., 30: 523-533. DOI: 10.1109/TMI.2010.2089383

Ricci, E. and R. Perfetti, 2009. Retinal blood vessel segmentation using line operators and support vector classification. IEEE Trans. Med. Imag., 28: 775785. PMID: 17948726

Sanchez, C.I., A. Mayo, M. Garcia, M.I. Lopez and R. Hornero, 2006. Automatic image processing algorithm to detect hard exudates based on mixture models. Proceedings of the IEEE 28th Annual International Conference of the Engineering in Medicine and Biology Society, Aug. 30-Sep. 3, IEEE Xplore Press, New York, pp: 4453-4456. DOI: 10.1109/IEMBS.2006.260434

Shobha, C. and R. Rajeshwari, 2014. Automatic fovea detection and grading of diabetic maculopathy severity levels. Int. J. Eng. Res. Tech.

Sopharak, A., B. Uyyanonvara and S. Barman, 2009. Automatic exudate detection from non-dilated diabetic retinopathy retinal images using fuzzy Cmeans clustering. J. Sens., 9: 2148-2161. DOI: $10.3390 / \mathrm{s} 90302148$

Walter, T., J. Klein, P. Massin and A. Erginay, 2002. A contribution of image processing to the diagnosis of diabetic retinopathy-detection of exudates in colour fundus images of the human retina. IEEE Trans. Med. Imag., 1: 1236-1243. PMID: 12585705 